\title{
Simulations of anisotropy and fabric development in polar ices
}

\author{
O. Castelnau and P. Duval \\ Laboratoire de Glaciologie et Géophysique de l'Environnement, CNRS, 38402 Saint-Martin-d'Hères Cedex, France
}

\begin{abstract}
The preferred orientation of ice crystals in polar ice sheets develops as a result of intracrystalline slip. Polycrystalline plasticity theory has been successfully used to simulate development of fabrics in rocks. In this study, we present a simple theory for plastic deformation and fabric evolution. Each crystal within the aggregate is assumed to deform only by basal glide, and recrystallization processes are not directly taken into account. We have adopted the uniform stress bound, that is, stress is supposed to be homogeneous in the polycrystal. Simulated fabrics in uniaxial compression and tension are similar to those observed in ice sheets. In simple shear, simulations show that $c$ axes rotate toward the direction of the maximal compression, in contradiction to the intuitive conclusion that stable orientations correspond to the easy glide position. But, these results are in agreement with predictions of Taylor theory and self-consistent approaches. We suggest that effects of vertical compression and/or rotation recrystallization are of great importance for fabric evolution in polar ice shects. The proposed model can reproduce viscosities of anisotropic ice samples tested in compression or tension but it is not able to reproduce the low viscosity of ices with a single-maximum fabric when tested in simple shear.
\end{abstract}

\section{INTRODUCTION}

Ice-flow modeling requires a constitutive relation giving the mechanical response of ice to stresses. Calculations described in most ice-sheet models are based on non-linear isotropic flow laws. Discrepancies between calculated and in-situ data can be due to deficiencies in the flow law (Van der Veen and Whillans, 1989). Anisotropic flow laws accounting for polar ice fabrics observed on deep ice cores have been proposed by Lliboutry and Duval (1985) and Van der Vcen and Whillans (1990). A simpler procedure accounting for anisotropic effects is to introduce an enhancement factor, defined as the ratio of the measured strain rates to that corresponding to isotropic ice (DahlJensen and Gundestrup, 1987). But this technique is not satisfactory since the flow law is always isotropic. Data from laboratory tests on Antarctic ices have already demonstrated the importance of anisotropy in the constitutive relations. For example, ice with $c$ axes aligned with the vertical axis is stiffer to horizontal normal stresses and softer with respect to shear stresses on horizontal planes (Pimienta and others, 1987).

Since fabrics change with time, the anisotropic constitutive equations must be associated with a model giving the evolution of the preferential orientation of $c$ axes. To develop such a model, deformation and recrystallization mechanisms must be determined. For conditions prevailing in polar ice sheets, intracrystalline dislocation glide is the main deformation mechanism (Pimicnta and Duval, 1987; Alley, 1992). Grain growth driven by grain-boundary energy is observed in the first hundred meters of central parts of the ice sheets. In warm ice, typically above $-12^{\circ} \mathrm{C}$, near to the bedrock, a recrystallization regime involving rapid migration of grain boundaries is observed. Multi-maxima fabrics are associated with this termed "migration recrystallization". Betwcen these two zones, new grains are formed during creep polygonization, and grain-boundary migration is driven by both grain-boundary and strain energies (Pimienta and Duval, 1989). As a result, grain-size generally does not increase. This recrystallization regime is termed "rotation recrystallization" (Poirier, 1990). The boundary between rotation and migration recrystallization regimes corresponds to a limited range of depths. Clear evidence of this transition was observed by Gow and Williamson (1976) at a depth of $1800 \mathrm{~m}$ in the Byrd Station ice core. An evolution from slow impuritycontrolled to fast impurity-frec grain-boundary migration, as proposed by Guillopé and Poirjer (1979), may be the origin of this rapid change of the recrystallization regime.

The purpose of this paper is to introduce a deformation model for the evolution of fabrics in polar ices. The effect of recrystallization is not taken into account. The formation mechanism of fabrics is based on the rotation of $c$ axcs induced by basal glide. The mechanical response of the polycrystal is obtained by an appropriate average of the response of each grain.

A first approach to this problem has been given by Azuma and Higashi (1985). The implicit assumption of their theory is that basal glide occurs at the same rate in each grain, regardless of its orientation. They found that the model predicts the observed fabric development at 
Dyc 3, Grecnland. This model, modified for uniaxial tension and pure shear, also describes fabrics observed in the Vostok ice core (Alley, 1988). But this kind of model has not been tested for simple shear or other strain configurations.

Considerable effort has been made to apply polycrystalline plasticity theory to rocks. The Taylor theory (Taylor, 1938), which assumes homogeneous deformation, has been successfully used to simulate fabric development as long as the plastic anisotropy of crystals is not too large. In viscoplastic self-consistent approaches (Molinari and others, 1987), which compromise between stress cquilibrium and strain continuity, each grain is considercd as an inclusion in a homogeneous isotropic medium, representing the "wcighted" avcrage of all grains. The interaction formula is solved by minimizing stress and strain-rate deviations. This theory can provide good results for materials with a small number of independent slip systems (Wenk and others, 1989, 1991).

Following Lliboutry and Duval (1985), the uniform stress approximation may be applicable to polar ice, since grain-boundary migration associated with grain growth or rotation recrystallization should accommodate intracrystalline dislocation glide. In this paper, the uniform stress bound (lower bound) is adopted to model both the creep and the development of fabrics in polar icc.

\section{THE MODEL}

Let $(\mathrm{O} x y z)$ be the reference frame of the laboratory (fixed) and $(\mathrm{O} \lambda \mu \nu)$ be the coordinate system of each crystal (crystallographic coordinate system), where $(\mathrm{O} \nu)$ corresponds to the $c$ axis (see Fig. 1). The Eulcr angles used are $\varphi$ and $\vartheta$. To bring into correspondence the set of axes $(\mathrm{O} x y z)$ with the set $(\mathrm{O} \lambda \mu \nu)$, two rotations are necessary: first, (Oxyz) is rotated by the angle $\varphi$ about the $y$ axis; then, the new system is rotated by the angle $\vartheta$ about the $z$ axis. A third Fuler angle is not nocessary, because there is no preferential slip direction in the basal plane.

Let $Q$ be the corresponding transformation matrix (expressed in $(\mathrm{O} \lambda \mu \nu)) ; Q$ is given by:

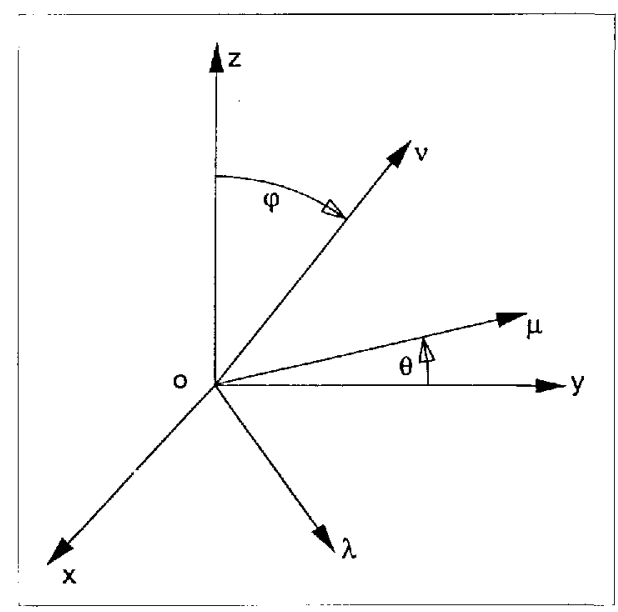

Fig. 1. Orientation of ice crystals defined in the crystal-

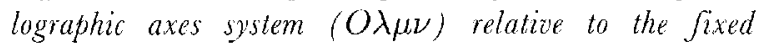
external axes $(O x y z)$. $(O \nu)$ corresponds to the $c$ axis. $(O \mu)$ is in the plane (Oxy).

$$
Q=\left(\begin{array}{ccc}
\cos \varphi \cdot \cos \vartheta & \cos \varphi \cdot \sin \vartheta & -\sin \varphi \\
-\sin \vartheta & \cos \vartheta & 0 \\
\sin \varphi \cdot \cos \vartheta & \sin \varphi \cdot \sin \vartheta & \cos \varphi
\end{array}\right)
$$

We call $T$ the average stress tensor applied to the polycrystal, expressed in (Oxyz). With the assumption of a uniform stress state, the stress tensor $T^{c}$ in cach grain is given, in the crystallographic axes, by:

$$
T^{\mathrm{C}}=Q \cdot \bar{T} \cdot Q^{\mathrm{T}}
$$

where $Q^{\mathrm{T}}$ is the transposcd matrix of $Q$.

We assume that monocrystals can deform only by basal glide. Hutchinson (1977) noted that the selfconsistent theory could provide reasonable estimates of the flow strength for hexagonal polycrystals displaving less than five independent slip systems. From Hutchinson, the overall deformation of polycrystalline ice is possible with only basal and prismatic slip. From Duval and others (1983), non-basal glide requires a stress at least 60 times larger than that required for basal slip at the same strain rate. The introduction of prismatic slip therefore has little effect on the deformation of ice crystals.

'Thus, only the stress components $T_{\lambda \nu}^{\circ}$ and $T_{\mu \nu}^{c}$ can induce basal glide.

The velocity gradient tensor $L^{\mathrm{c}}$ for each crystal, expressed in $(\mathrm{O} \lambda \mu \nu)$, is given by:

$$
L^{c}=\left(\begin{array}{ccc}
0 & 0 & L_{\lambda \nu}^{c} \\
0 & 0 & L_{\mu \nu}^{c} \\
0 & 0 & 0
\end{array}\right)
$$

With a viscoplastic power law for basal glide

$$
L_{\lambda \nu}^{c}=A \cdot \tau^{n-1} \cdot T_{\lambda \nu}^{c} \quad \text { and } \quad L_{\mu \nu}^{c}=A \cdot \tau^{n-1} \cdot T_{\mu \nu}^{c}
$$

where

$$
\tau=\sqrt{T_{\lambda \nu}^{\mathrm{c} 2}+T_{\mu \nu}^{\mathrm{c}}{ }^{2}}
$$

A simple calculation shows that, in our deformation model, the exponent $n$ is necessarily the same for the bulk sample and the monocrystal. Simulations were done using two values of the stress exponent $n$. The value $n=1.5$ was chosen from data obtained by Pimienta and others (1987) on polycrystalline ice at low stresses. The value $n=3$ was taken with reference to the commonly adopted value for polycrystalline ice.

The coefficient $A$ is fitted to reproduce the viscosity of an isotropic ice sample; we have found, in order to reproduce the Glen law coefficient $B o$ at $-15^{\circ} \mathrm{C}$,

$$
\begin{aligned}
& \text { for } n=3 \text { : } \\
& A=1.4 \times 10^{-24} \mathrm{~Pa}^{-3} \mathrm{~s}^{-1} \text {, which gives } \\
& B o=3.2 \times 10^{-25} \mathrm{~Pa}^{-3} \mathrm{~s}^{-1} \text { from Legac, } \\
& \text { 1980); } \\
& \text { for } n=1.5 \text { : } \\
& A=5.6 \times 10^{-17} \mathrm{~Pa}^{-1.5} \mathrm{~s}^{-1} \text {, which gives } \\
& B o=1.9 \times 10^{-17} \mathrm{~Pa}^{-1.5} \mathrm{~s}^{-1} \text { (from Pimienta }
\end{aligned}
$$

It is important to compare these values with the coefficient of the power law obtained using mechanical 
tests on a monocrystal, even if the exponent of the flow law is different (Duval and others, 1983). For a single crystal, strain rates for basal glide are at least two orders of magnitude higher than those determined using the above coefficients. In a polycrystalline aggregate, grainboundary migration and or recrystallization should accommodate strain incompatibilities (Lliboutry and Duval, 1985) but not enough to impede completely grain interactions.

The expression of the velocity-gradient tensor $L$ of each grain in the laboratory axes is:

$$
L=Q^{\mathrm{T}} \cdot L^{\mathrm{C}} \cdot Q
$$

which can be decomposed into the sum of the strain-rate tensor $D$ symmetric) with the rotation-rate tensor $W$ (antisymmetric):

$$
L=D+W
$$

with

$$
D=\left(L+L^{\mathrm{T}}\right) / 2 \text { and } W=\left(L-L^{\mathrm{l}}\right) / 2 .
$$

The bulk velocity gradient $\bar{L}$ (in Oxyz) of the sample is given by averaging all tensors $L$ :

$$
\bar{L}=\frac{1}{m} \sum_{1}^{m} L
$$

where $m$ is the number of grains.

$\bar{L}$, in the same way as $L$, can be decomposed as:

$$
\bar{L}=\ddot{D}+\ddot{W} \text {. }
$$

Now we apply the boundary conditions: let $\bar{L}_{\mathrm{f}}$ be the final average velocity gradient of the polycrystal after applying the boundary conditions (with the decomposition: $\bar{L}_{\mathrm{f}}=\bar{D}_{\mathrm{f}}+\bar{W}_{\mathrm{f}}$, and $L_{\mathrm{f}}$ the final velocity gradient of each grain (with: $L_{\mathrm{f}}=D_{\mathrm{f}}+W_{\mathrm{f}}$ ), both expressed in (Oxyz).

We make the assumption that during the transformation, the rotation of the matrix of material containing the considered grain is equal to the bulk final imposed rotation $\bar{W}_{\mathrm{f}}$. In the most rigorous way, the rotation of this matrix has to be taken equal to the average rotation of the direct environment of the inclusion (in our case about fivc to ten grains). The assumption made here does not allow us to calculate local effects concerning the crystal orientations but it does not affect significantly the average fabric evolution. Furthermore, the rotational part $W_{\mathrm{f}}$ of the final velocity gradient of each grain has to be equal to the rotation of the matrix, i.e.

$$
W_{\mathrm{f}}=\bar{W}_{\mathrm{f}} .
$$

Let $\bar{W}_{\text {be }}$ be the rotation ratc that is necessary to add to the average velocity gradient $\bar{L}$ to satisfy the boundary conditions:

$$
\bar{L}_{\mathrm{f}}=\bar{L}+\bar{W}_{\mathrm{b} c}
$$

i.e.

$$
\bar{D}_{\mathrm{f}}=\bar{D} \quad \text { and } \quad \bar{W}_{\mathrm{f}}=\bar{W}+\bar{W}_{\mathrm{bc}} .
$$

In the case of irrotational transformations (irrotational uniaxial compression, tension or pure shear), $\bar{W}_{\mathrm{bc}}$ is given by:

$$
\bar{W}_{\mathrm{bc}}=-\bar{W} \text {. }
$$

In the case of a simple shear in the plane $(O x y)$, in the dircction $(\mathrm{O} x)$ :

$$
\bar{W}_{\mathrm{bc}}=\left(\begin{array}{ccc}
0 & 0 & \bar{D}_{x z} \\
0 & 0 & 0 \\
-\bar{D}_{x z} & 0 & 0
\end{array}\right)-\bar{W} .
$$

Now let $W_{\text {bc }}$ be the rotation rate that is necessary to add to the velocity gradient $L$ to satisfy the local boundary conditions. The final velocity gradient of each grain is then given by:

$$
L_{\mathrm{f}}=L+W_{\mathrm{bc}},
$$

i.e.

$$
D_{\mathrm{f}}=D \quad \text { and } \quad W_{\mathrm{f}}=W+W_{\mathrm{bc}} .
$$

Equations (3) and (5) then give:

$$
W_{\mathrm{bc}}=\bar{W}_{\mathrm{f}}-W
$$

The tensor $W_{\mathrm{bc}}$ then corresponds to the rotation rate of the $c$ axis of the considered grain.

The calculation stcp used for the simulations is $1 \%$ equivalent strain, which is defined as:

$$
\epsilon_{\mathrm{eq}}=\sum \dot{\epsilon}_{\mathrm{cq}} \cdot \mathrm{d} t
$$

where $\mathrm{d} t$ is the time step, and $\dot{\epsilon}_{\mathrm{eq}}$ is the cquivalent strain rate, defined as:

$$
\dot{\epsilon}_{\mathrm{eq}}=\sqrt{\frac{2}{3} \bar{D}_{\mathrm{f}}: \bar{D}_{\mathrm{f}}}
$$

This calculation step is small enough to consider all tensors constant during one step.

The gradient transformation tensor $F_{6}$ corresponding to the $c$-axis rotation is given by:

$$
F_{\mathrm{c}}=\exp \left(\mathrm{d} t \cdot W_{\mathrm{bc}}\right)
$$

where the exponential function is defined, for secondorder tensors, with the relation:

$$
\exp (A)=I+A+\frac{A^{2}}{2 !}+\ldots+\frac{A^{n}}{n !}+\ldots
$$

with $I$ the identity tensor.

The final $c$-axis orientation is given by the vector $C_{\mathrm{f}}$ (expressed in Oxyz), with:

$$
C_{\mathrm{f}}=F_{\mathrm{c}} . C
$$

where $C$ is the unit vector of the $(\mathrm{O} \nu)$ axis in $(\mathrm{O} x y z)$

$$
C=\left(\begin{array}{c}
\sin \varphi \cdot \cos \vartheta \\
\sin \varphi \cdot \sin \vartheta \\
\cos \varphi
\end{array}\right)
$$

In the same way, the transformation gradient tensor $\bar{F}$ of 
the bulk sample, corresponding to the considered calculation step, is given by:

$$
\bar{F}=\exp \left(\mathrm{d} t \cdot \bar{L}_{\mathrm{f}}\right)
$$

and the total (cumulative) transformation gradient tensor $F_{\mathrm{t}}$ by:

$$
\bar{F}_{\mathrm{t}}=\vec{F} \cdot \bar{F}_{0}
$$

wherc $\vec{F}_{0}$ is the initial transformation of the polycrystal.

Results of this model can then be separated in two parts:

Equation (6) gives the fabric development

Equation (4) gives the rheological law of the anisotropic ice sample.

\section{RESULTS AND DISCUSSION}

\subsection{Fabric evolution}

Figure 2 displays the simulated fabrics in (a) uniaxial compression, (b) uniaxial extension, (c) pure shear and (d) simple shear, respectively, at 45\%,30\%, 50\% and $40 \%$ equivalent strain. All these fabrics were simulated with $n=1.5$ and with a constant applied stress. However, for simple shear, it is necessary to impose axial stresses increasing with strain to impede axial deformation, particularly when the preferential orientation of $c$ axes becomes pronounced. The corresponding evolution of the normalized equivalent strain rate is given in Figure 3. As the fabric develops, basal glide bccomes impossible and,

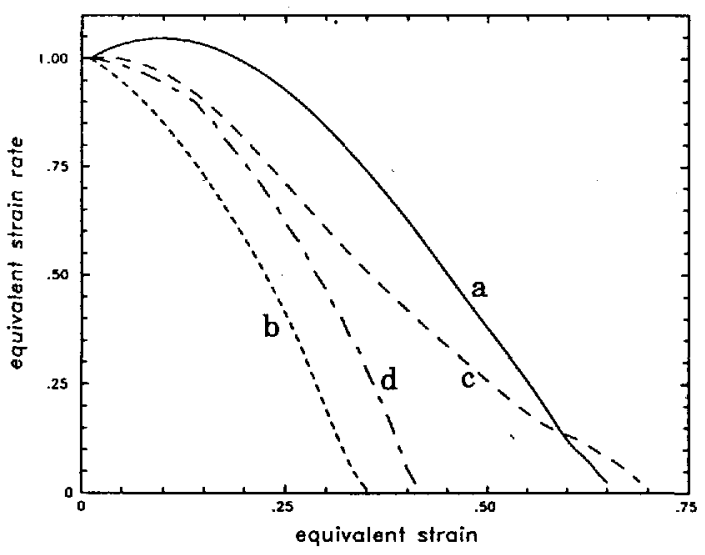

Fig. 3. Evolution of the normalized equivalent strain rate with the equivalent strain obtained by simulation in: (a) uniaxial compression; (b) uniaxial extension; (c) pure shear; (d) simple shear. $\epsilon_{\mathrm{eq}}=0$ corresponds to a randomly oriented aggregate. $n=1.5$.

in the framework of our model, deformation of the polycrystal cannot occur any more.

Fabric patterns in compression, extension and pure shear are in agreement with those simulated by Azuma and Higashi (1985) and Alley (1988), and with fabrics observed in ice sheets, e.g. Dye 3 (Herron and others, 1985) and Vostok (Lipenkov and others, 1989) cores.

In simple shear, the $c$ axes rotate toward the direction of the maximal compression. This behavior is contrary to the intuition that the slip plane orients itself into the shear plane. Strong single-maximum fabrics observed in several deep ice cores are attributed to the rotation of the $c$ axis

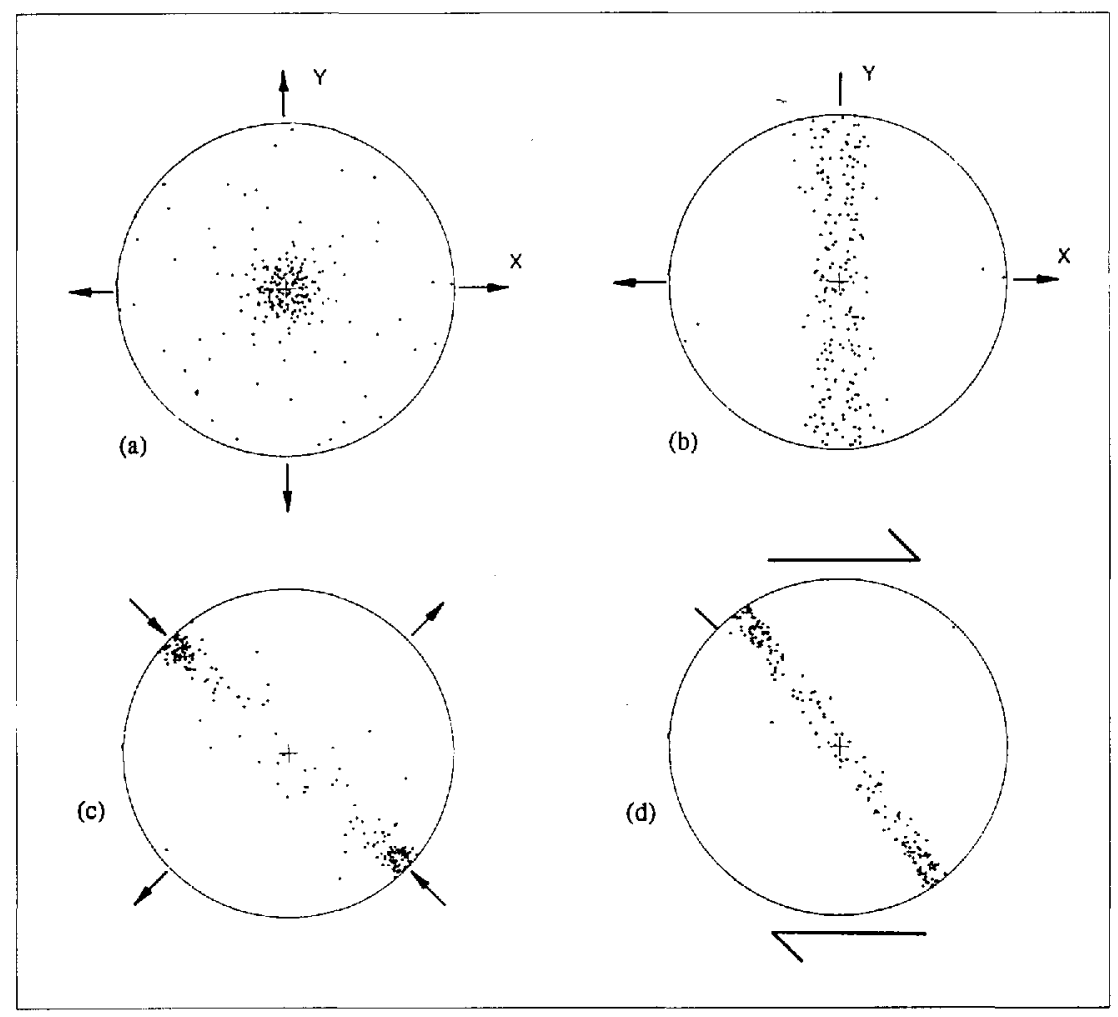

Fig. 2. Simulated c-axes fabric diagrams for an initially isotropic ice sample deformed: (a) to $45 \%$ equivalent strain in uniaxial compression; (b) to $30 \%$ in uniaxial tension; (c) to 50\% in pure shear and (d) $1040 \%$ in simple shear; arrozes denote the sense of deformations; calculations were done with the exponent of the flow lawe $n=1.5 ; 200$ grains. 
by shear, and basal planes are parallel to the permanent shear plane (Alley, 1988).

It is interesting to compare these simulated fabrics with those obtained by the viscoplastic self-consistent theory on anisotropic rocks. The simulations for peridotite by Wenk and others (1991) gave results similar to those obtained above for ice, and especially for peridotites containing 30-100\% enstatite. Enstatite has, as for ice, a single easy-slip plane.

For $\alpha$-quartz, for which basal slip predominates, the $c$ axis fabric diagrams in simple shear deduced from both the Taylor and self-consistent theories (Wenk and others, 1989) are qualitatively similar to those obtained using the homogeneous stress bound.

Therefore, strong single-maximum fabrics cannot be attributed to simple shear alone. But, a metastable orientation of the $c$ axis along the normal to the permanent shear plane is found with the homogeneous stress bound. This orientation can be made stable if a compression normal to the macroscopic shear plane is imposed. These conditions are found in ice sheets; a more or less constant vertical strain rate is associated with the imposed horizontal shear stress (Dahl-Jensen, 1989).

Another explanation of the strong single-maximum fabrics is to associate rotation of basal planes by dislocation glide and rotation recrystallization. Such an explanation was given by Kamb (1972) and.Bouchez and Duval (1982). A review of mechanisms for the development of fabrics in ice sheets was recently given by Paterson (1991).

\subsection{Rheological parameters of the flow law}

As seen in section 2, the model is able to produce the crolution of the rheological parameters of a polycrystalline aggregate, corresponding to the imposed stress state (or strain history).

Experimental results from uniaxial compression, biaxial compression and torsion tests on ice samples from the Vostok and Law Dome (Antarctica) ice cores have been analyzed by Pimienta and others (1987). Values of the rheological parameters at $-15^{\circ} \mathrm{C}$, corresponding to the anisotropic power law given by Lliboutry and Duval (1985), were determined.

We call $B$ the coefficient of the extended Glen law for anisotropic ice corresponding to an imposed deformation, and Bo the coefficient of the Glen law for isotropic ice.
Table 1 gives the ratios $B / B O$ for three different deformation configurations: uniaxial compression in direction $\left(z^{\prime} z\right)$, uniaxial compression in direction $\left(x^{\prime} x\right)$ and simple shear parallel to $\left(x^{\prime} x\right)$, where the axis system $(x y z)$ is shown in Figure 2. These ratios are compared with those deduced from the homogeneous stress model with an exponent $n=3$. The input fabrics are those given by the model with simulations in uniaxial compression $\left(\epsilon_{\mathrm{eq}}=45 \%\right)$ and uniaxial extension $\left(\epsilon_{\mathrm{eq}}=30 \%\right.$ ) (see Fig. 2), using 200 grains. The value of $B o$ is that obtained with a randomly oriented aggregate (isotropic).

Comparison of these results shows that our model is able to reproduce with quite good fidelity the different viscosities of both ice samples tested in compression.

Data from laboratory tests were obtained at high stresses for which the exponent $n$ is equal to 3 . The basic assumption for the homogeneous stress theory is probably not well adapted since high internal stresses must be present at high stresses (Duval and others, 1983). But experimental data from mechanical tests on anisotropic samples at low stresses (about 0.01-0.05 MPa) are not available.

Both ratios $B / B o$ obtained for the Law Dome sample in simple shear expcriments are quite different by a factor of 4). The simulated value cannot be higher than 4.3 (simulation with a single crystal where $\varphi=0$ ), whereas a value of about 10 is obtaincd from laboratory tests on ice with a strong single-maximum fabric (Pimienta and others, 1987; Budd and Jacka, 1989).

Thesc discrepancies probably originatc from the value of the coefficient $A$ of the flow law of ice crystals, fitted to reproduce the viscosity of isotropic polycrystalline ice. From Lliboutry and Duval (1985), this parameter must depend on the fabric. The viscosity of ice crystals inserted into an anisotropic aggregate with a strong singlemaximum fabric is probably lower than that associated with an isotropic ice.

\section{ACKNOWLEDGEMENTS}

This work has been funded by the Centre National de la Recherche Scientifique (CNRS, France). We are very grateful to G. Canova for fruitful discussions.

Table 1. Experimental data (from Pimienta and others, 1987) and simulated values of the ratios $B / B o$, where $B$ is the flow-law coefficient for Vostok $(-2039 \mathrm{~m})$ and Law Dome anisotropic ices and Bo is the average coefficient for isotropic ice samples (from Legac, 1980). Fabrics taken for simulations are given in Figure $2 a$ and b; the external axes are represented in Figure $2 ; n=3$

\begin{tabular}{lcccc}
\hline$B / B o$ & \multicolumn{2}{c}{ Law Dome } & \multicolumn{2}{c}{ Vostok $(-2039 \mathrm{~m})$} \\
& Experimental & Simulation & Experimental & Simulation \\
& & & & \\
\hline Compression $\left(z^{\prime} z\right)$ & 0.4 & 0.39 & 0.7 & 0.93 \\
Compression $\left(x^{\prime} x\right)$ & 0.19 & 0.30 & 0.06 & 0.03 \\
Simple shear $\left(x^{\prime} x\right)$ & 10 & 2.74 & 0.7 & 1.43 \\
& & & &
\end{tabular}




\section{REFERENCES}

Alley, R.B. 1988. Fabrics in polar ice shects: development and prediction. Science, 240 4831 , 493-495.

Alley, R.B. 1992. lilow-law hypotheses for ice-sheet modeling. f. Glaciol., 38 (29), 24.j-256.

Azuma, N. and A. Higashi. 1985. Formation processes of icc fabric pattern in ice sheets. Amm. Gilaciol, 6, $130 \cdot 131$.

Bouches, J.l. and P. Duval. 1982. The fabric of polycrustalline ice deformed in simple shear: experiments in torsion, natural deformation and geometrical interpretation. Texures and Microstructures, 5, 171-190.

Budd, W. F'. and 'T. H. Jacka. 1989. A revicw of ice rheology for ice sheet modelling. Cold Reg. Sci. Terhnot, 16 2;, 107-144.

Dahl-Jensen, D. 1989. Steady thermomechanical flow along twodimensional flow lines in large grounded ice sheets. .7. Geophys. Res., $94(B 8), 10,35.5-10,362$

Dahl-Jensen, D, and N.S. Gundestrup. 1987. Constitutive properties of icc at Dye-3, Greenland. Inlernational Association of Hydrological Sciences Fublication 170 , Symposium at Vancouver 1987 The Physical Basis of Tee Sheet Modelling), 31-43.

Duval, P., M.F. Ashby and I. Anderman. 1983. Rate-controlling processes in the creep of polycrystalline ice. J. Phy. Chem., 87 (21), $4066-4074$

Gow, A.J. and T. Williamson. 1976. Rheological implications of the intemal structure and crystal fabrics of the West Antarctic ice sheet as revaled by deep core drilling at Byrd Station. CRREL Rep. 76-35.

Guillopé, M. and J. P. Poiricr. 1979. Dynamic recrystallization during creep of single-crystalline halite: an experimental study. 7. Geophys. Res., 84 B $10,55575567$.

Herron, S. L., C. C. Langway, Ir and K. A. Brugger. 1985. Ultrasonic velocitics and erystalline anisotropy in the ice core from Dyc 3 , Greenland. In Langway, C. C., Jr, H. Oeschger and W. Dansgatard, eds. Grepnland ire core: geophysics, geochemistry, and the enironment. Washington, DC, American Goophysical Cnion, 23-31. Geophysical Monograph 33.)

Hufchinson, J. W. 1977. Creep and plasticity of hexagonal polycrystals as related to single crystal slip. Metall. Trans. 8A 9), 1465-1469.

Kamb, W. B. 1972. Experimental recrystallization of ice under stress. In Heard. H. C., Y. Borg, N. L. Carter and C. B. Raleigh, eds. Floz' and fratwe of rocks. Washington, DC, American Geophysical Union, 211-241. (Geophysical Monograph 16.)

LcGac, H. 1980. Contribution à la détermination des lois de comportement de la glace polycristalline (anélasticité et plasticité).
Thése de $3^{r}$ rycle, Lniversité Srientifique et Médicale de Grenoble. Lipenkov, V.Ya., N.I. Barkov, P. Duwal and P. Pinitenta. 1989 Crystalline texture of the $2083 \mathrm{~m}$ ice core at lostok Station. Antarctica. 7. Glaciol. 35 (121), 392-398.

Lliboutry, L. and P. Juval. 1985. Various isotropic and anisotropic ices found in glaciers and polar ice caps and their corresponding rhcologies, Annales Geophysicae, 3 2$\}, 207224$.

Molinari, A., G.R. Canova and S. Ahzi. 1987. A self consistent approach of the large deformation polycrystal viscoplasticity. Acta Metall, 35 12), 29832994.

Paterson, W. S. B. 1991. Why ice-age ice is sometimes "soft". Cold Rrg. Sci. Tectinol, $\mathbf{2 0} 1), 75-98$.

Pimienta, P. and P. Duval. 1987. Rate controlling processes in the creep of polar glacier ice. 7. Phys. (Paris), 48, Colloq. C1, 243.248. (Supplément au 3.)

Pimienta, P. and P. Duval. 1989. Rheology of polar glacier ice Abstract. Ann. Glaciol. 12, 206207.

Pimienta, P., P. Duval and Y. Ya. Lipenkov. 1987. Mechanical behavior of anisotropic polar ice. Internationat Association of Hytrologicat Sriences Publication 170 Symposium at Vancouver $1987-$ - The Phosical Basis of Ice Sheet Modelling), 5766.

Poirier, J.P. 1990. Creep of enstals. Cambridge, Cambridge University Press.

Taylor, G. I. 1938. Plastic strain in metals. f. Inst. Met. 62, 307-324.

Van der Vecn, G.J. and I. M. Whillans. 1989. Forec budget: II Application to two-dimensional flow along Byrd Station Strain Vetwork, Antarctica. ff. Glaciol, 35 (119), 61-67.

Van der Veen, C.J. and I. M. Whillans. 1990. Flow laws for glacier ice: comparison of numerical predictions and ficld measurements. 7. Glaciol., 36 124), 324-339.

Wenk, H.-R., G. Canova, A. Molinari and L.F. Kocks. 1989. Viscoplastic modelling of texture development in quartzitc. 7. Geophys. Res., 94(B12), 17,895-17,906.

Wenk. H.-R, K. Bennett. G. R. Canova and A. Molinari. 1991. Modelling plastic deformation of peridotite with the self-consisten $t$ theory. 7. Geophys. Res., 96(B5), 8337--8349.

The accuracy of references in the text and in this list is the responsibility of the authors, to whom queries should be addressed. 